\title{
État des connaissances sur les céphalophes (genres Cephalophus et Philantomba) des forêts denses humides d'Afrique centrale (synthèse bibliographique)
}

\author{
Fructueux G. A. Houngbégnon ${ }^{(1)}$, Bonaventure Sonké ${ }^{(2)}$, Cédric Vermeulen ${ }^{(1)}$, \\ Jean-Louis Doucet ${ }^{(1)}$ \\ (1) Université de Liège - Gembloux Agro-Bio Tech. Terra Teaching and Research Centre. Forest is life. Passage des Déportés, \\ 2. BE-5030 Gembloux (Belgique). E-mail : fructueux.houngbegnon@yahoo.fr \\ (2) Université de Yaoundé I. École Normale Supérieure. Département de Biologie. Laboratoire de Botanique systématique et \\ d’Écologie. BP 047 Yaoundé (Cameroun).
}

Reçu le 26 mars 2018, accepté le 28 janvier 2019, mis en ligne le 12 mars 2019.

Cet article est distribué suivant les termes et les conditions de la licence CC-BY (http://creativecommons.org/licenses/by/4.0/ deed.fr)

Introduction. Cet article propose une synthèse des connaissances actuelles sur la classification, l'écologie et la biologie des céphalophes des forêts denses humides d'Afrique centrale (genres Cephalophus et Philantomba). Il fait aussi le point sur les pressions anthropiques subies par ces mammifères terrestres.

Littérature. De nombreuses publications sur les céphalophes vivant dans les forêts denses humides d'Afrique centrale se focalisent sur la chasse, qu'elle soit de subsistance ou commerciale, y compris le braconnage. La classification taxonomique des céphalophes ayant été récemment révisée, de nouvelles espèces ont été décrites, alors que leur écologie n'est que très peu documentée. Cette synthèse met en exergue les lacunes en termes de connaissances scientifiques et propose les thématiques prioritaires pour de futures recherches.

Conclusions. Bien que de nombreux domaines restent à explorer pour certaines espèces (utilisation de l'habitat, domaine vital, etc.), il est suggéré d'étudier en priorité les techniques de différenciation des espèces de céphalophes lors des inventaires, leur rôle dans la dynamique forestière et l'impact de l'exploitation forestière sur leurs populations.

Mots-clés. Bovidae, antilopes, ruminants, chasse, forêts, fruit, régénération forestière, Afrique centrale.

State of knowledge on duikers (genera Cephalophus and Philantomba) of Central Africa rainforests. A review Introduction. This article provides a synthesis of current knowledge on classification, ecology and biology of duikers living in central African rainforests (genera Cephalophus and Philantomba). It also provides an update on the anthropogenic pressures on these terrestrial mammals.

Literature. Many publications on central African duikers are focused on subsistence or commercial hunting, and poaching. As the taxonomic classification of duikers has recently been revised, new species have been described with very little information available on their ecology. This synthesis highlights the gaps in the scientific knowledge and proposes priority themes for future research.

Conclusions. Although many aspects remain to be explored for some species (i.e., habitat use, home range), it is suggested to investigate in priority: the inventory methods, the role of duikers in forest dynamics, and the impact of logging on their populations.

Keywords. Bovidae, antelopes, ruminants, hunting, forests, fruit, forest regeneration, Central Africa.

\section{INTRODUCTION}

Les céphalophes sont des antilopes endémiques des forêts et des savanes de l'Afrique subsaharienne (Feer, 1988 ; Colyn et al., 2010 ; Kingdon, 2013).
Ils appartiennent à l'ordre des Cetartiodactyles, à la famille des Bovidés et à la sous-famille des Antilopinés. Ils forment l'un des groupes de mammifères les plus homogènes des points de vue anato-morphologique, géographique et écologique (Dubost, 1980 ; Feer, 
1988). Les populations de céphalophes représentent une part considérable de la biomasse animale dans les écosystèmes forestiers (Vanthomme et al., 2010). Leur régime alimentaire est composé principalement de fruits (frugivores) et de feuilles (folivores) dont la composition semble varier selon les saisons (Feer, 1989a). Les céphalophes font partie des espèces les plus chassées (FAO, 2010) et constituent à ce titre une source importante de protéines et de revenus par le biais de la vente de leur viande (Poulsen et al., 2009 ; Fargeot, 2013). Cette chasse a un impact important sur de nombreuses espèces (Delvingt et al., 2001 ; Laurance et al., 2006), y compris sur celles considérées jadis comme résilientes à la pression de chasse (Grande-Vega et al., 2016).

Cet article a pour objectif de faire un état des connaissances sur les céphalophes (genres Cephalophus et Philantomba). Il ambitionne d'être le plus synthétique possible sur les céphalophes des forêts denses humides d'Afrique centrale. Les aspects suivants sont plus particulièrement abordés :

- les relations phylogénétiques entre taxons, leur taxonomie et leur répartition géographique,

- leurs caractéristiques biologiques,

- l'utilisation de l'espace,

- les pressions anthropiques.

Les recherches documentaires ont été réalisées en utilisant principalement les bases de données Google Scholar, Scopus, BASE (Bielefeld Academic Search Engine) et ORBI (Open Repository and Bibliography). Les mots-clés suivants ont été utilisés en français et en anglais : céphalophes, chasse, exploitation forestière, forêt dense humide, densité, domaines vitaux, dissémination des graines, régénération forestière, inventaire, phylogénie, Afrique centrale.

La combinaison des mots-clés dans les différentes bases de données a permis de recenser plus de 3000 références. De ces résultats, une sélection des publications a été faite sur base des titres et des résumés, ce qui a permis de retenir dans un premier temps 78 articles. Après lecture, 19 articles ont encore été écartés soit parce qu'ils étaient moins informatifs, soit parce qu'ils n'étaient pas suffisamment centrés sur la zone d'intérêt de cette synthèse, permettant de retenir finalement 59 articles.

\section{RELATIONS PHYLOGÉNÉTIQUES, TAXONOMIE ET RÉPARTITION GÉOGRAPHIQUE}

Les relations phylogénétiques entre taxons de la tribu des Cephalophini font l'objet d'un débat intense à cause des liens taxonomiques non résolus entre espèces sœurs (Ntie et al., 2010 ; Johnston \& Anthony, 2012). Le plus vieux fossile de Cephalophini connu à ce jour daterait de 5,8 Ma(Vrba,1995) et selon Castello (2016), l'existence de cette tribu remonterait à $12 \mathrm{Ma}$. Autrefois, le genre Philantomba était considéré comme un synonyme du genre Cephalophus. Cependant, sur base d'analyses cladistiques, Grubb \& Groves (2001) l'ont considéré comme un genre séparé, proche du genre Sylvicapra (Cephalophini). Le nombre d'espèces de céphalophes existants dans les forêts d'Afrique reste encore sujet à controverse. En effet, une nouvelle espèce a encore récemment été décrite (Colyn et al., 2010). De plus, des taxons jadis classés comme des sous-espèces, sur base de descriptions morphologiques ou biologiques, ont récemment été élevés au rang d'espèces grâce à de nouvelles analyses phylogénétiques (Groves et al., 2011; Castello, 2016). Ainsi, dans les forêts denses humides d'Afrique centrale, on peut distinguer 6 espèces du genre Philantomba et 11 espèces du genre Cephalophus (Groves et al., 2011 ; Castello, 2016).

Par ailleurs, d'autres études organisent les céphalophes en trois groupes phylogénétiques distincts (van Vuuren \& Robinson, 2001 ; Johnston \& Anthony, 2012). On retrouve donc dans les forêts denses humides d'Afrique centrale :

- les céphalophes nains (six espèces),

- les céphalophes rouges (neuf espèces),

- les grands céphalophes (deux espèces).

\subsection{Les céphalophes nains}

Ce groupe est constitué exclusivement des espèces du genre Philantomba:

- Philantomba aequatorialis (Cephalophus

aequatorialis Matschie),

- Philantomba anchietae (Cephalophus anchietae

Bocage),

- Philantomba congica (Cephalophus nyasae congicus Lönnberg),

- Philantomba defriesi (Cephalophus nyasae defriesi Rothschild),

- Philantomba melanorhea (Cephalophus melanorheus Gray),

- Philantomba simpsoni (Cephalophus simpsoni

Thomas).

Grubb \& Groves (2001) les organisent en deux sous-groupes: le sous-groupe à pattes grises (dont P. aequatorialis, P. congica, P. melanorhea), situé au nord de l'aire de répartition du genre et le sousgroupe à pattes rouges (dont P. anchietae, P. defriesi, $P$. simpsoni), situé au sud de l'aire de répartition (Figure 1).

Ces espèces se discriminent facilement du genre Cephalophus par leur morphologie générale caractérisée par une petite taille $(30-43 \mathrm{~cm})$, un faible poids $(3,8-10 \mathrm{~kg})$ et une coloration grise ou 
1. Philantomba melanorhea

2. Philantomba congica

3. Philantomba aequatorialis

4. Philantomba simpsoni

5. Philantomba anchietae

6. Philantomba defriesi

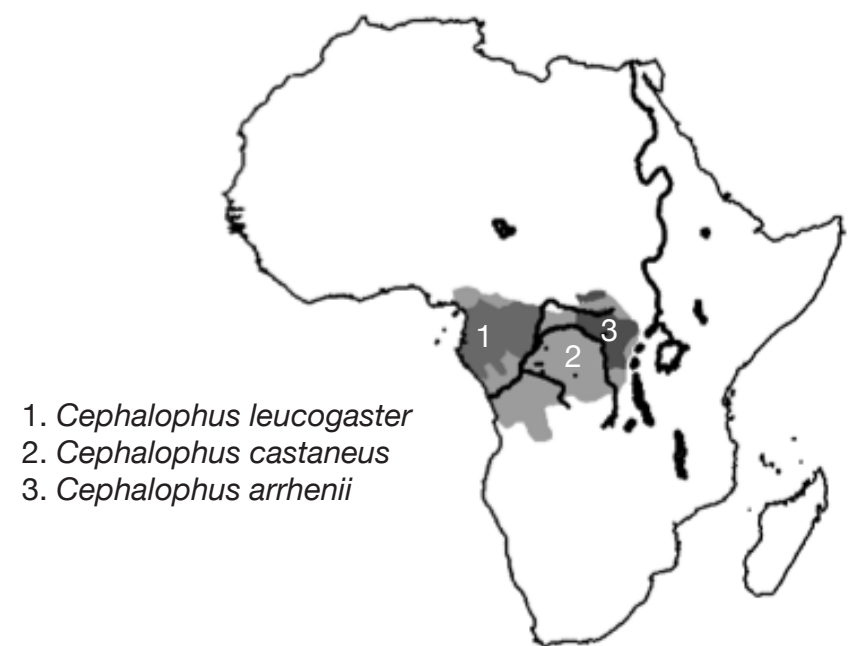

1. Cephalophus silvicultor

2. Cephalophus nigrifrons

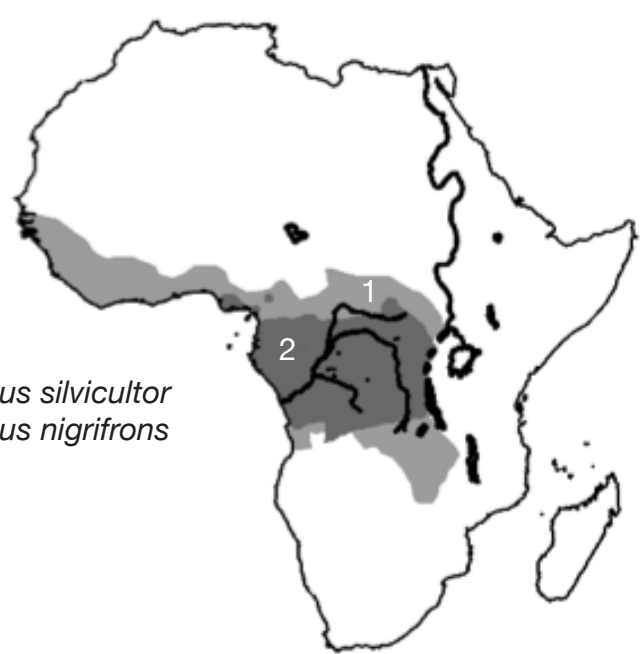

Figure 1. Répartition géographique des espèces de céphalophes présentes dans les forêts denses humides d'Afrique centrale. Carte adaptée d'après les données de Groves et al. (2011) - Distribution range of duikers of central Africa rainforest. Map adapted from data of Groves et al. (2011).

gris-brun (Dubost, 1980 ; Kingdon, 2013 ; Castello, 2016). Les analyses phylogénétiques soutiennent fortement la monophylie du genre Philantomba et sa position basale par rapport aux autres céphalophes (van Vuuren \& Robinson, 2001 ; Johnston \& Anthony, 2012). Cependant, à l'intérieur du genre, les espèces formeraient, selon les auteurs, soit des associations paraphylétiques (Johnston \& Anthony, 2012), soit monophylétiques (Colyn et al., 2010).

\subsection{Les céphalophes rouges}

Ce groupe monophylétique comprend neuf espèces :

- Cephalophus arrhenii (Cephalophus castaneus arrhenii Lönnberg),

- Cephalophus callipygus Peters,

- Cephalophus crusalbum (Cephalophus ogilbyi crusalbum Grubb),
- Cephalophus hypoxanthus (Cephalophus nigrifrons hypoxanthus Grubb et Groves),

- Cephalophus johnstoni Thomas,

- Cephalophus leucogaster Gray,

- Cephalophus nigrifrons Gray,

- Cephalophus ogilbyi Waterhouse,

- Cephalophus weynsi Thomas.

Ces espèces sont très variables en termes de coloration (orange, brun, rouge ou noir), taille $(40-60 \mathrm{~cm})$ et poids (14-25 kg) (Groves et al., 2011 ; Kingdon, 2013). Leur distinction est parfois difficile et s'appuie sur un nombre limité de traits physiques (couleur du pelage, des pattes, du front, du dos, de la croupe, forme de la tête et des cornes). Les espèces sœurs, sympatriques en Afrique centrale, C.callipygus et C.ogilbyi, forment un groupe paraphylétique remettant en cause leur statut d'espèce (Johnston \& Anthony, 2012). 


\subsection{Les grands céphalophes}

Ce groupe comprend deux grands céphalophes (jusqu'à $80 \mathrm{~cm}$ et $80 \mathrm{~kg}$ ) dont les colorations sont typiques : Cephalophus silvicultor (Antilope sylvicultrix Afzelius, brun-noir avec des poils érectiles jaunes formant une figure triangulaire au bas du dos), Cephalophus castaneus (Cephalophus dorsalis castaneus Thomas, brun-rouge avec une large bande noire allant du haut de la tête à la queue) (Kingdon, 2013).

Toutefois, faisant fi de ces classifications, les espèces de céphalophes sont souvent réparties, pour des raisons pratiques, en trois catégories en fonction de la couleur de leur pelage :

- les céphalophes bleus (regroupant tout le genre Philantomba),

- les céphalophes rouges (regroupant tout le genre Cephalophus à l'exception de C. silvicultor),

- le céphalophe à dos jaune (C. silvicultor) (Fimbel et al., 2000 ; van Vliet \& Nasi, 2007).

Les différents groupes de céphalophes utilisés dans la suite de cette contribution font référence à ces dernières catégories.

La distribution et les traits distinctifs de l'ensemble de ces espèces sont repris respectivement dans les figure 1 et annexe 1.

En somme, malgré les révisions de Groves et al. (2011), Johnston \& Anthony (2012) et Castello (2016), les relations phylogénétiques de plusieurs espèces de céphalophes restent à préciser.

\section{CARACTÉRISTIQUES BIOLOGIQUES}

\subsection{Régime alimentaire}

Les céphalophes sont d'importants consommateurs primaires terrestres (Feer, 1989a). Ils sont considérés principalement comme des frugivores (jusqu'à $90 \%$ de leur régime alimentaire) et des folivores, les proportions relatives et la composition de leur régime alimentaire variant avec les saisons (Feer, 1989a). Les céphalophes digèrent efficacement les tissus végétaux ingérés grâce à un appareil digestif comprenant un estomac à trois chambres. Les études antérieures sur leur écologie ont montré qu'ils se nourrissent également de bourgeons, de graines, de champignons et occasionnellement d'autres animaux (Kingdon \& Hoffmann, 2013). Les études de Dubost (1984) sur les contenus stomacaux de plusieurs espèces de ruminants (dont C.callipygus, C.castaneus, C.leucogaster, $C$. nigrifrons, $C$. silvicultor et $P$. congica) au nord-est du Gabon montrent que seulement trois à sept espèces de fruits sont communes (Annexe 2). Cela s'expliquerait par différents types d'habitats préférentiels. Une recherche similaire réalisée dans la même zone par Feer (1989a) a révélé que $86 \%$ à $89 \%$ du poids sec des aliments ingérés par $C$. callipygus et $C$. castaneus était composé de fruits. L'auteur démontre également que $C$. castaneus qui est nocturne est moins frugivore que C.callipygus. En outre, la consommation de feuilles (majoritairement vertes) est moins diversifiée que celle des fruits chez les céphalophes (Feer, 1989a). Cephalophus nigrifrons est l'espèce la plus folivore, probablement parce qu'il fréquente les biotopes inondés où les fruits sont moins abondants (Feer, 1989a). Enfin, C.callipygus et C.castaneus consomment parfois d'autres animaux, tels que les petits arthropodes (fourmis, termites, myriapodes) pour le premier et des rongeurs (Atherurus africanus Gray) ou des termites, pour le second (Feer, 1989a). Cependant, il semblerait que $C$. castaneus consomme plus de matières animales que $C$. callipygus (Feer, 1989a).

L'annexe 2 illustre différentes espèces végétales dont les fruits sont consommés par les céphalophes. Les familles les plus appétées sont Annonaceae, Burseraceae, Euphorbiaceae, Fabaceae, Myristicaceae, Rubiaceae et Sapotaceae.

$\mathrm{Au}$ total, l'importance des fruits dans le régime alimentaire des populations de céphalophes est bien documentée, celle des aliments secondaires (feuilles, champignons, matières animales, etc.) l'est moins.

\subsection{Dispersion des graines par les céphalophes}

La consommation de fruits prédispose les céphalophes à être de potentiels disséminateurs de graines.

La capacité de dispersion des graines dépend de plusieurs facteurs. D'après Gautier-Hion et al. (1985), la couleur des fruits ne semble pas être un facteur déterminant pour plusieurs espèces de céphalophes, même si elles sont diurnes et peuvent distinguer les couleurs. En outre, Illius \& Gordon (1992) signalent que la taille des céphalophes impose des contraintes quant à la sélection des fruits. Ainsi, C. silvicultor pourrait logiquement avaler de plus grosses graines que $P$. congica. Dubost (1984) a démontré à ce sujet que les plus petites espèces de céphalophes n'avalent pas les fruits de plus de $3 \mathrm{~cm}$ de diamètre, alors que les plus grandes peuvent en avaler qui atteignent $6 \mathrm{~cm}$ de diamètre. Toutefois, la finesse de la mastication, associée au processus long et complexe de la digestion, prédisposerait les céphalophes à être de mauvais agents de dissémination (Newing, 2001). Une bonne mastication et une excellente digestion impliquent en effet une transformation significative de l'état des produits ingérés et donc, une faible probabilité que des graines viables se retrouvent dans les fèces. Cependant, Alexandre (1982) a montré la présence dans des échantillons de fèces de Cephalophus niger Gray (absent d'Afrique centrale) de graines provenant 
principalement de Solanum verbascifolium L. et de Musanga cecropioides R.Br. ex Tedlie. Des graines de petite taille pourraient donc échapper au processus de mastication et de digestion.

Les fèces ne sont pas les seuls canaux par lesquels les graines peuvent être dispersées. Les céphalophes peuvent également disséminer les graines en les recrachant loin du semencier, lors de la rumination dans leur lieu de repos (Gautier-Hion et al., 1985). Dans ce dernier cas, la dureté du tégument séminal serait le principal facteur qui conditionnerait la viabilité des graines recrachées. La détermination des graines que les céphalophes peuvent rejeter lors de la rumination peut se faire soit en analysant le contenu de leur rumen, soit par des observations en captivité (Feer, 1995).

L'annexe 3 présente des données sur la taille des graines consommées par quatre espèces de céphalophes et le rôle potentiel de celles-ci dans la dispersion de ces graines.

On peut conclure de tout ce qui précède que l'étude du caractère disperseur ou prédateur des graines par les céphalophes reste un vaste champ d'investigation.

\subsection{Reproduction}

Les informations sur la reproduction de plusieurs espèces de céphalophes sont encore lacunaires. Les espèces du genre Philantomba sont les seules qui vivent en couple (Dubost, 1980). Les autres espèces seraient plutôt solitaires, à l'exception des périodes de reproduction. Des cas de polygynie ont été observés chez $C$. callipygus et $C$. castaneus et le domaine vital du mâle se superpose alors à celui de deux femelles (Feer, 1988). La copulation est généralement précédée d'une parade nuptiale au cours de laquelle le mâle poursuit la femelle. Les mâles peuvent être, à ce moment, très agressifs envers d'autres animaux (Castello, 2016).

Il semblerait que la maturité sexuelle des mâles du genre Philantomba soit atteinte entre 12 et 18 mois, alors que les femelles seraient matures entre 9 et 12 mois. La durée de la gestation serait de sept mois. La portée serait généralement d'un jeune (rarement deux), sevré vers cinq mois. La longévité serait d'une douzaine d'années. Dans le genre Cephalophus, les différentes valeurs seraient légèrement supérieures : 12 à 18 mois et 9 à 30 mois respectivement pour la maturité sexuelle des mâles et des femelles, jusqu'à neuf mois de gestation et 12 à 22 ans de longévité. La période de sevrage pourrait cependant descendre à 3,5 mois pour certaines espèces (Groves et al., 2011 ; Kingdon \& Hoffmann, 2013 ; Castello, 2016). Kranz \& Lumpkin (1982) ont par ailleurs observé que les jeunes de $C$. silvicultor élevés en captivité étaient sevrés à l'âge d'un mois et pouvaient déjà consommer de l'herbe à l'âge de huit jours. Les naissances chez les céphalophes s'effectuent tout au long de l'année, bien que des pics peuvent survenir selon les espèces. Au nord-est du Gabon, Dubost \& Feer (1992) ont observé des pics de naissance lors des périodes plus sèches (juillet et janvier) chez $P$. congica, alors que la situation semble beaucoup moins claire pour C. castaneus et C. callipygus.

Enfin, il est important de noter que les données sur la reproduction des céphalophes sont pour la plupart issues de l'étude d'animaux en captivité. Elles ne peuvent donc être généralisées aux animaux vivant dans leur milieu naturel.

\section{UTILISATION DE L'ESPACE}

\subsection{Types d'habitat}

En Afrique centrale, jusqu'à sept espèces de céphalophes peuvent vivre en sympatrie (Newing, 2001). Les espèces $C$.callipygus, $C$.castaneus et le groupe des céphalophes bleus sont les plus fréquemment observés et donc les plus étudiés (Dubost, 1980 ; Dubost, 1984 ; Feer, 1989a ; Feer, 1989b ; Dubost \& Feer, 1992 ; Feer, 1995). Les céphalophes bleus occupent des habitats très variés, y compris les mosaïques forestières et les forêts secondaires; toutefois, les grands espaces ouverts sont évités (Dubost, 1980 ; Castello, 2016). Ils sont territoriaux et vivent en petits groupes formés d'un mâle, d'une femelle et d'un ou de deux petits (Dubost, 1980 ; Tutin et al., 1997 ; Mockrin, 2009). Cephalophus arrhenii, C.callipygus, C.castaneus, C. leucogaster, C.ogilbyi et $C$.weynsi vivent plutôt dans les forêts matures ou dans les vieilles forêts secondaires (Kingdon \& Hoffmann, 2013 ; Castello, 2016). Cependant, à l'instar de C. hypoxanthus et C. johnstoni, C. ogilbyi peut se retrouver à des altitudes élevées telles que les forêts de montagne. Cephalophus nigrifrons préfère les forêts inondées et les marécages. Il possède à cet effet des sabots longs et plats adaptés à ce type d'environnement (Dubost, 1984; Groves et al., 2011). Cephalophus silvicultor a une occupation éparse de son aire de distribution. Il est généraliste au niveau de l'habitat, mais se retrouve souvent à la lisière des forêts et des savanes comme C.crusalbum (Kranz \& Lumpkin, 1982 ; Groves et al., 2011).

D'une manière générale, les habitats préférentiels de la plupart des espèces de céphalophes sont documentés. Néanmoins, des progrès restent à faire quant à leur utilisation.

\subsection{Domaine vital et rythme d'activité}

L'étude du domaine vital des céphalophes n'a été effectuée de façon approfondie que sur un nombre limité d'espèces. Leur domaine vital peut recouvrir des types de végétation différents. Les céphalophes témoignent 
d'une fidélité particulière à un site, mais l'étendue de leur domaine vital peut varier pour une même espèce en fonction du sexe, de l'âge et des saisons (Feer, 1988). La variation saisonnière du domaine vital est notamment tributaire de la disponibilité en fruits. Ainsi, au cours des saisons sèches, une légère augmentation de la taille du domaine vital est perceptible suite à la recherche de fruits dans ces périodes de moindre disponibilité (Feer, 1988). C'est particulièrement le cas chez les plus grandes espèces qui ont besoin d'importantes ressources énergétiques. Le domaine vital des céphalophes bleus serait en moyenne de 6 ha, ne variant ni en fonction de l'âge, ni du sexe (Mockrin, 2009), ni des saisons (Dubost, 1980). Celui des céphalophes rouges oscillerait entre 7 et 80 ha (Feer, 1988 ; Groves et al., 2011). Plus spécifiquement, les tailles des domaines vitaux des femelles $C$. callipygus et $C$. castaneus ainsi que du mâle $C$. callipygus seraient très similaires ( $40 \mathrm{ha})$, alors que le mâle $C$. castaneus aurait un domaine vital deux fois plus grand ( $~ 80 \mathrm{ha})$. Cephalophus silvicultor aurait le plus large domaine vital, autour de 200 ha (Feer, 1988).

Pour ce qui est des rythmes d'activité, Dubost (1980) et Feer (1988) concluent respectivement que $P$. congica et $C$.callipygus seraient principalement actifs entre $6 \mathrm{~h}$ et $10 \mathrm{~h}$ et $15 \mathrm{~h}$ et $19 \mathrm{~h}$. À l'inverse, C. castaneus présenterait un rythme nycthéméral opposé avec des périodes d'activités s'étalant de $19 \mathrm{~h}$ à $22 \mathrm{~h}$ et de $2 \mathrm{~h}$ à 6 h (Groves et al., 2011). Ainsi, C. callipygus serait diurne, alors que $C$. castaneus serait nocturne, ce qui permettrait une ségrégation temporelle des activités entre les deux espèces (Feer, 1989b). Cephalophus silvicultor serait quant à lui plus actif à l'aube et au crépuscule, bien qu'il soit aussi observable de façon intermittente à d'autres moments, de jour comme de nuit (Kingdon \& Hoffmann, 2013).

On peut conclure de ces différentes recherches que l'étude du domaine vital et du rythme d'activité de certaines espèces de céphalophes sont encore lacunaires.

\subsection{Densités}

Les densités des trois groupes de céphalophes (bleus, rouges, à dos jaune) seraient respectivement comprises entre 0,9 à 78 individus $\cdot \mathrm{km}^{-2}, 0,3$ à 19 individus $\cdot \mathrm{km}^{-2}$ et 0,3 à 2,15 individus $\cdot \mathrm{km}^{-2}$ (Dubost, 1980 ; Tutin et al., 1997 ; Hart, 2000 ; Groves et al., 2011).

Toutefois, l'estimation des effectifs des céphalophes en forêt dense humide tropicale et la mesure de la densité et des biomasses qui en découlent restent des questions controversées, tant l'écart entre les différentes estimations est important. Delvingt et al. (2001) notent un facteur d'écart de 1 à 17 entre les estimations de biomasse reprises dans neuf études différentes. Cette variabilité s'expliquerait par le recours aux méthodes d'inventaires (diurne ou nocturne) par transect linéaire avec comptage des indices de présence directs et indirects tels que White (1994) le préconise. Étant donné que les céphalophes sont difficilement observables dans les forêts denses, ce sont les indices indirects (fèces notamment) qui sont les plus utilisés. Les estimations qui en résultent, généralement calculées avec le logiciel Distance (Thomas et al., 2010), sont sujettes à des biais (Viquerat et al., 2013) liés aux aptitudes des observateurs à détecter les indices (van Vliet \& Nasi, 2007) et à l'utilisation de taux de défécation et de vitesse de dégradation des fèces (Plumptre, 2000). En effet, la vitesse de dégradation peut changer en fonction des facteurs stationnels et saisonniers. Les taux de défécation peuvent aussi varier selon les zones et les espèces en fonction des ressources nutritives disponibles. Par exemple, Koster \& Hart (1988), en République Démocratique du Congo, ont estimé le taux de défécation des céphalophes rouges et des céphalophes bleus respectivement à 4,4 et 4,9 fèces par jour et la vitesse de dégradation de leurs fèces respectivement à 21 et 18 jours. Au Gabon, White (1994) a évalué la vitesse de décomposition des fèces des céphalophes rouges à 4,3 jours en saison pluvieuse et deux mois en saison sèche. Dans le même pays, mais dans un autre milieu, van Vliet et al. (2009) déterminent une vitesse de dégradation des fèces de 3,4 jours pendant la saison sèche et 0,7 jour pendant la saison pluvieuse. Ces auteurs démontrent par ailleurs que les coléoptères (surtout nocturnes) jouent un rôle prépondérant dans la vitesse de décomposition des fèces. Ces divers constats peuvent conduire à une surestimation de la densité des céphalophes en saison sèche. À ceci, il faut ajouter la difficulté d'associer les fèces à la bonne espèce de céphalophes. En conséquence, les chercheurs utilisent généralement les trois groupes de céphalophes précités au regard de la taille des fèces (Koster \& Hart, 1988 ; van Vliet \& Nasi, 2007), entrainant davantage d'imprécisions dans les estimations.

Lors des inventaires directs, outre la densité de la végétation, la détectabilité des céphalophes dépend également des pressions cynégétiques qui modifient leur comportement. Lahm (1993) a démontré que les céphalophes adoptent, dans les zones chassées, un comportement cryptique en réponse aux observateurs qui approchent. Cependant, Croes et al. (2006) ont noté des observations contraires et signalent un comportement stationnaire des céphalophes dans les zones non chassées et une fuite souvent accompagnée de cris dans les terroirs de chasse. En conséquence, Viquerat et al. (2013) estiment que les inventaires directs diurnes ne sont fiables que sous une pression de chasse contrôlée.

Plusieurs études ont tenté de réduire les biais, soit en génotypant les fèces (van Vliet et al., 2007 ; 
Ntie et al., 2010), soit en se basant exclusivement sur des comptages directs lors d'inventaires nocturnes, lesquels ont démontré leur capacité à augmenter considérablement les taux de rencontre (Robinson et al., 2016). Enfin, le récent développement des inventaires par pièges photographiques laisse entrevoir des estimations à l'avenir plus fiables (Nakashima et al., 2018).

Des progrès dans ce domaine sont à espérer. Toute tentative de gestion durable des prélèvements de céphalophes doit passer à un moment ou à un autre par une précision des méthodes d'inventaires.

\section{PRESSIONS ANTHROPIQUES}

\subsection{Pression cynégétique}

Les références trouvées dans le cadre de cette synthèse sont plutôt axées sur la chasse, notamment la chasse de subsistance, la chasse commerciale et le braconnage. Nous allons ici volontairement nous limiter à quelquesunes d'entre elles.

Différents outils de chasse sont utilisés pour la capture des céphalophes : les fusils, les pièges à cou ou à patte (Delvingt et al., 2001) et les filets, bien que ceux-ci tendent à disparaitre (Fargeot, 2013). Les estimations de la consommation annuelle de la viande de chasse dans le bassin du Congo peuvent aller jusqu'à cinq millions de tonnes (Brown, 2003). Les céphalophes sont reconnus comme les premiers produits de chasse et représentent à ce titre une part importante des prélèvements tant en nombre qu'en biomasse (Hart, 2000 ; Mockrin, 2009 ; Yasuoka et al., 2015). Ils peuvent totaliser jusqu'à $80 \%$ des prélèvements de chasse (Vanthomme et al., 2010).

Selon Newing (2001), la pression de chasse sur les populations de céphalophes a probablement atteint un niveau insoutenable. Les céphalophes bleus apparaissent comme étant les plus prélevés et ont été longtemps considérés comme les plus résilients à la pression cynégétique à cause de leur caractère anthropophile (Yasuako et al., 2015). De plus, ils sont réputés prolifiques avec un cycle de reproduction assez court (FAO, 2010). Ainsi, selon certaines études, la pression de chasse n'aurait que peu d'influence sur la démographie des céphalophes bleus (Mockrin, 2009 ; Hart \& Kingdon, 2013). Cette assertion a toutefois été remise en question par Grande-Vega et al. (2016).

La durabilité de la chasse dépendrait de la maitrise du prélèvement maximum soutenable $\left(P_{R R}\right)$ sur chaque terroir de chasse. Robinson \& Redford (1991) ont proposé un modèle pour le calculer.

$$
P_{R R}=f P_{\max }, P_{\text {max }}=D\left(\lambda_{\max }-1\right)
$$

avec $P_{R R}$ : prélèvement maximum soutenable exprimé en individu $\cdot \mathrm{km}^{-2} \cdot \mathrm{an}^{-1} ; D$ : densité exprimée en individus. $\mathrm{km}^{-2} ; \lambda_{\max }$ : croissance démographique annuelle maximale exprimée en individus $\cdot \mathrm{an}^{-1} ; P_{\max }$ : production annuelle maximale exprimée en individus $\cdot \mathrm{km}^{-2} \cdot \mathrm{an}^{-1} ; f$ : facteur variant en fonction de la longévité des espèces. Selon Robinson \& Redford (1991), $f$ prend la valeur de 0,$2 ; 0,4$; ou 0,6 respectivement pour les animaux à longévité supérieure à dix ans, comprise entre cinq et dix ans, et inférieure à cinq ans.

Le tableau 1 synthétise les valeurs des différents paramètres disponibles dans la littérature pour le calcul du $P_{R R}$. On peut noter une différence de $30 \%$ entre les valeurs de $\lambda_{\text {max }}$ observées pour le groupe des céphalophes bleus, une différence de $25 \%$ pour le groupe des céphalophes rouges et de $10 \%$ pour le céphalophe à dos jaune. Les valeurs de $f$ sont de 0,4 pour les céphalophes bleus et rouges, et de 0,2 pour le céphalophe à dos jaune.

Yasuoka (2006) estime, pour les forêts denses humides d'Afrique centrale, un prélèvement maximum soutenable à une valeur comprise entre 0,03 et 1 individu $\cdot \mathrm{km}^{-2} \cdot \mathrm{an}^{-1}$ pour les céphalophes bleus et entre 0,07 et $0,2 \mathrm{individu} \cdot \mathrm{km}^{-2} \cdot \mathrm{an}^{-1}$ pour le céphalophe à dos jaune. Ces calculs, fondés sur la production durable maximale, sont cependant considérés par van Vliet \& Nasi (2008) comme peu fiables, en raison notamment des incertitudes évoquées précédemment quant à l'estimation des densités.

Les céphalophes sont souvent considérés comme de bons indicateurs du niveau de défaunation du fait

Tableau 1. Données pour le calcul du prélèvement maximum soutenable (d'après le modèle de Robinson \& Redford, 1991) - Data for maximum sustainable harvest (based on the model of Robinson \& Redford, 1991).

\begin{tabular}{lllll}
\hline \multirow{2}{*}{ Groupe d'espèces } & \multicolumn{2}{l}{$\lambda_{\max }$ (individus·an $^{-1)}$} & \multicolumn{1}{c}{$\mathbf{f}$} \\
\cline { 2 - 4 } & Fimbel et al. (2000) & Fa et al.(1995) & Feer (1993) & Yasuoka (2006) \\
\hline Céphalophes bleus & 1,63 & 1,63 & 2,33 & 0,4 \\
Céphalophes rouges & 1,24 & 1,54 & 1,65 & 0,4 \\
Céphalophe à dos jaune (Cephalophus silvicultor) & - & 1.54 & 1,39 & 0,2 \\
\hline
\end{tabular}

$\lambda_{\max }$ : croissande démographique annuelle maximale - maximum annual population growth; $\mathrm{f}$ : facteur variant en fonction de la longévité des espèces - factor varying according to the longevity of the species. 
de leurs niveaux de résiliences variables (Nasi \& van Vliet, 2011). Yasuoka et al. (2015) proposent de calculer le ratio entre les nombres (chassés ou inventoriés) de céphalophes bleus et de céphalophes rouges. Ces auteurs considèrent que, dans une zone à pression anthropique faible, le ratio céphalophes bleus/céphalophes rouges est faible. Cependant, lorsque toutes les espèces de céphalophes sont rares, ce qui témoigne d'une forte pression anthropique, l'interprétation du ratio devient délicate.

Il ressort de toutes ces études que la pression cynégétique a un impact considérable sur les populations de céphalophes et que le calcul des taux d'extraction reste sujet à controverse.

\subsection{Impacts de l'exploitation forestière}

Constituant la principale forme d'affectation des terres dans le bassin du Congo, l'exploitation du bois d'œuvre est sélective, avec une moyenne d'un pied abattu par ha tous les 25 à 30 ans (Nasi et al., 2012). En l'absence de contrôle du réseau routier dont l'ouverture est indispensable à l'exploitation, on observe généralement une recrudescence du braconnage. En conséquence, il n'est guère aisé de discriminer les impacts directs de l'exploitation de ceux de la chasse. En outre, en l'absence d'alternatives, l'exploitation forestière contribue à créer des concentrations humaines qui engendrent une demande en viande de brousse accrue et des filières pour y répondre, dans lesquelles les céphalophes constituent une biomasse considérable. À titre d'exemple, Semeki et al. (2014) démontrent que dans une concession forestière en République Démocratique du Congo, les céphalophes représentaient $55 \%$ de toute la biomasse sauvage consommée.

D'après Laurance et al. (2006), la présence de routes dans les forêts aurait un impact important sur les populations de céphalophes. De même, Poulsen et al. (2011) notent que les forêts exploitées, même en absence d'activités de chasse, ne maintiendraient pas les mêmes densités de céphalophes que les forêts non exploitées, bien que Clark et al. (2009) démontrent que les espèces réagissent différemment. Par contre, il n'y aurait pas un effet direct de l'exploitation forestière sur la communauté de céphalophes tant à court terme (4 ans) qu'à long terme (25 ans) (White, 1992).

Il est possible d'inférer de ces études que l'impact de l'exploitation forestière sur les différentes espèces de céphalophes (en dehors de celle de la chasse provoquée par l'ouverture des routes) reste mal connu.

\section{CONCLUSIONS}

À l'issue de cette synthèse, il s'avère que les relations phylogénétiques entre les différentes espèces de céphalophes demeurent peu résolues, malgré les révisions récentes proposées par Groves et al. (2011), Johnston \& Anthony (2012) et Castello (2016).

Les recherches menées sur le régime alimentaire des céphalophes montrent que ce sont des espèces majoritairement frugivores (jusqu'à $90 \%$ de leur régime alimentaire). Toutefois, l'importance des aliments secondaires consommés (feuilles, champignons, matières animales, etc.) est peu documentée. Par ailleurs, bien que la zoochorie joue un rôle déterminant dans la dynamique des forêts (Vanthomme et al., 2010), il existe un déficit d'informations sur la dissémination des graines par les céphalophes.

Quant aux informations sur la biologie de la reproduction des céphalophes, elles ne concernent que quelques espèces et sont pour la plupart issues de l'observation d'animaux en captivité. Elles ne peuvent donc pas être généralisées aux animaux vivant en liberté.

Les méthodes actuelles d'inventaires, reposant majoritairement sur des indices indirects, ne peuvent fournir que des informations approximatives sur les densités et les biomasses. De même, l'utilisation de l'habitat par les populations de céphalophes reste peu établie.

Les pressions cynégétiques sur la communauté de céphalophes soulignent le niveau insoutenable qu'a atteint leur chasse. En outre, les études sur le prélèvement maximum soutenable sont sujettes à polémique et ne peuvent être généralisées à toutes les forêts denses humides d'Afrique centrale. Enfin, quant à l'impact de l'exploitation forestière, il est difficilement séparable de l'impact de la chasse.

Néanmoins, cette synthèse bibliographique permet une meilleure compréhension des différents enjeux afférents à ces espèces et constitue de ce fait un outil d'aide à la décision dans la mise en place des politiques de gestion et de protection des céphalophes dans les écosystèmes forestiers d'Afrique centrale.

Pour de futures recherches, nous suggérons que les thématiques suivantes soient prioritairement abordées : - le rôle des céphalophes dans la dispersion des graines et la régénération forestière,

- l'impact de l'exploitation forestière sur les populations de céphalophes,

- la distinction des différentes espèces de céphalophes lors des inventaires par des techniques innovantes (génotypage des fèces et pièges photographiques).

\section{Remerciements}

Les auteurs adressent leurs remerciements au FRS-FNRS pour la bourse doctorale FRIA accordée à Fructueux G.A. Houngbégnon et au «Programme de Promotion de l'Exploitation Certifiée des Forêts » pour son appui financier dès l'entame de cette recherche. L'étude a également 
bénéficié de l'appui de la société Pallisco et de l'ASBL Nature+. Les auteurs témoignent leur reconnaissance à tout le personnel du centre de recherche TERRA de l'Université de Liège - Gembloux Agro-Bio Tech, notamment l'équipe de la CARE Forest is life.

\section{Bibliographie}

Alexandre D.Y., 1982. La dispersion de Solanum verbascifolium en Côte d'Ivoire : rôle des céphalophes. Rev. Ecol., 36, 293-295.

Brown D.,2003.Bushmeat \& poverty alleviation: implication for development policy, https://www.odi.org/sites/odi. org.uk/files/odi-assets/publications-opinion-files/3308. pdf, (19/05/2017).

Castello J.R., 2016. Bovids of the world. Antelopes, gazelles, cattle, goats, sheep, and relatives. Princeton, NJ, USA; Oxford, UK: Princeton University Press.

Clark C.J., Poulsen J.R., Malonga R. \& Elkan Jr P.W., 2009. Logging concessions can extend the conservation estate for central African tropical forests. Conserv. Biol., 23(5), 1281-1293.

Colyn M. et al., 2010. Discovery of a new duiker species (Bovidae: Cephalophinae) from the Dahomey Gap, West Africa. Zootaxa, 2637, 1-30.

Croes B.M. et al., 2006. The influence of hunting on antipredator behavior in Central African monkeys and duikers. Biotropica, 39(2), 257-263.

Delvingt W., Dethier M., Auzel P. \& Jeanmart P., 2001. La chasse villageoise Badjoué, gestion coutumière durable ou pillage de la ressource gibier? In: Delvingt W., éd. La forêt des hommes. Terroirs villageois en forêt tropicale africaine. Gembloux, Belgique: Les Presses agronomiques de Gembloux, 65-92.

Dubost G., 1980. L'écologie et la vie sociale du céphalophe bleu (Cephalophus monticola Thunberg), petit ruminant forestier africain. Z. Tierpsychol., 54, 205-266.

Dubost G., 1984. Comparison of the diets of frugivorous forest ruminants of Gabon. J. Mammal., 65(2), 298-316.

Dubost G. \& Feer F., 1992. Saisons de reproduction des petits ruminants dans le nord-Gabon, en fonction des variations des ressources. Mammalia, 56(1), 25-43.

Fa J.E., Juste J., Perez del Val J. \& Castroviejo J., 1995. Impact of market hunting on mammal species in Equatorial Guinea. Conserv. Biol., 9, 1107-1015.

FAO, 2010. Prise en compte de la biodiversité dans les concessions forestières d'Afrique centrale. Document de travail sur la biodiversité forestière, $n^{\circ} 1$. Rome : FAO.

Fargeot C., 2013. La chasse commerciale en Afrique centrale: une menace pour la biodiversité ou une activité économique durable? Le cas de la république centrafricaine. Thèse de doctorat: Université Paul Valéry, Montpellier (France).

Feer F., 1988. Stratégie écologique de deux espèces de bovidés sympatriques de la forêt sempervirente africaine
(Cephalophus callipygus et $\mathrm{C}$. dorsalis) : influence $d u$ rythme d'activité. Thèse de doctorat : Université Pierre et Marie Curie, Paris (France).

Feer F., 1989a. Comparaison des régimes alimentaires de Cephalophus callipygus et C.dorsalis, bovides sympatriques de la forêt sempervirente africaine. Mammalia, 53, 563-604.

Feer F., 1989b. The use of space by 2 sympatric duikers (Cephalophus callipygus and Cephalophus dorsalis) in an African rain-forest - The role of activity rhythms. Rev. Ecol . Terre Vie, 44, 225-248.

Feer F., 1993. The potential for sustainable hunting and rearing of game in tropical forests. In: Hladik C.M. et al., eds. Tropical forests, people and food. Paris: Unesco/ MAB, 691-708.

Feer F., 1995. Seed dispersal in African forest ruminants. J. Trop. Ecol., 11(4), 683-689.

Fimbel C., Curran B. \& Usongo L., 2000. Enhancing the sustainability of duiker hunting through community participation and controlled access in the Lobéké region of southeastern Cameroon. In: Robinson J.G. \& Bennett E.L., eds. Hunting for sustainability in tropical forest. New York, NY, USA: Colombia University Press, 356-374.

Gautier-Hion A. et al., 1985. Fruit characters as a basis of fruit choice and seed dispersal in a tropical forest vertebrate community. Oecologia, 65, 324-337.

Grande-Vega M., Farfán M.A., Ondo A. \& Fa J.E., 2016. Decline in hunter offtake of blue duikers in Bioko Island, Equatorial Guinea. Afr. J. Ecol., 54, 49-58.

Groves C.P. et al., 2011. Family Bovidae (hollow-horned ruminants). In: Wilson D.E. \& Mittermeier R.A. Handbook of the mammals of the world. Vol. 2. Hoofed Mammals. Barcelona, Spain: Lynx Edicions, 755773.

Grubb P. \& Groves C.P., 2001. Revision and classification of the Cephalophinae. In: Wilson V.J., ed. Duikers of Africa: masters of the African forest floor. Bulawayo, Zimbabwe: Chipangali Wildlife Trust, 703-728.

Hart J.A., 2000. Impact and sustainability of indigenous hunting in the Ituri forest, Congo-Zaire: a comparison of hunted and unhunted duiker populations. In: Robinson J.G. \& Bennett E.L., eds. Hunting for sustainability in tropical forests. New York, NY, USA: Columbia University Press, 106-155.

Hart J.A. \& Kingdon J., 2013. Philantomba monticola blue duiker. In: Kingdon J. \& Hoffmann M., eds. The mammals of Africa. Volume VI: pigs, hippopotamuses, chevrotain, giraffes, deer and bovids. London: Bloomsbury, 228-234.

Illius A.W. \& Gordon I.J., 1992. Modelling the nutritional ecology of ungulate herbivores-evolution of body size and competitive interactions. Oecologia, 89(3), 428-434.

Johnston A.R. \& Anthony N.M., 2012. A multi-locus species phylogeny of African forest duikers in the subfamily Cephalophinae: evidence for a recent radiation in the Pleistocene. BMC Evol. Biol., 12, 120. 
Kingdon J., 2013. The Kingdon field guide to African mammals. London: Bloomsbury Publishing.

Kingdon J. \& Hoffmann M., 2013. Mammals of Africa. Volume VI: Pigs, hippopotamuses, chevrotain, giraffes, deer and bovids. London: Bloomsbury Publishing.

Koster S. \& Hart J., 1988. Methods of estimating ungulate populations in tropical forests. Afr. J. Ecol., 26, 117-126.

Kranz K.R. \& Lumpkin S., 1982. Notes on the yellowbacked duiker Cephalophus sylvicultor in captivity with comments on its natural history. Int. Zoo Yearbook, 22, 232-240.

Lahm S.A., 1993. Ecology and economics of human/wildlife interaction in Northeastern Gabon. PhD Thesis: New York University, New York (USA).

Laurance W.F. et al., 2006. Impacts of roads and hunting on central African rainforest mammals. Conserv. Biol., 20(4), 1251-1261.

Mockrin H.M., 2009. Duiker demography and dispersal under hunting in Northern Congo. Afr. J. Ecol., 48, 239-247.

Nakashima Y., Fukasawa K. \& Samejima H., 2018. Estimating animal density without individual recognition using information derivable exclusively from camera traps. Afr. J. Ecol., 55, 735-744.

Nasi R. \& van Vliet N., 2011. Mesure de l'abondance des populations d'animaux sauvages dans les concessions forestières d'Afrique centrale. Unasylva, 238(62), 49-55.

Nasi R., Billand A. \& van Vliet N., 2012. Managing for timber and bioversity in the Congo Basin. For. Ecol. Manage., 268, 103-111.

Newing H., 2001. Bushmeat hunting and management: implications of duiker ecology and interspecific competition. Biodivers. Conserv., 10, 99-118.

Ntie S., Soto-Calderon I.D., Eaton M.J. \& Anthony N.M., 2010. Cross-species amplification of bovid microsatellites in central African duikers (genus Cephalophus) and other sympatric artiodactyls. Mol. Ecol. Resour., 10, 10591065.

Plumptre A.J., 2000. Monitoring mammal populations with line transect techniques in African forests. J. Appl. Ecol., 37, 356-368.

Poulsen J.R., Clark C.J., Mavah G. \& Elkan P.W., 2009. Bushmeat supply and consumption in a tropical logging concession in Northern Congo. Conserv. Biol., 23, 15971608.

Poulsen J.R., Clark C.J. \& Bolker B.M., 2011. Decoupling the effects of logging and hunting on an Afrotropical animal community. Ecol. Appl., 21, 1819-1836.

Robinson C.A.J., Zollner P.A. \& Kpanou J.-B., 2016. Night and day: evaluating transect methodologies to monitor duikers in the Dzanga-Sangha protected areas, central African Republic. Afr. J. Ecol., 55, 222-232.

Robinson J.G. \& Redford K.H., 1991. Sustainable harvest of neotropical forest mammals. In: Robinson J.G. \& Redford K.H., eds. Neotropical wildlife use and conservation. Chicago, Il, USA: University of Chicago Press, 415-429.
Semeki N.J., Belani M.J., Ntoto M.R. \& Vermeulen C., 2014. Consommation de produits d'origine animale dans la concession forestière 039/11 de la SODEFOR à Oshwe (R.D. Congo). Tropicultura, 32(3), 147-155.

Thomas L. et al., 2010. Distance software: design and analysis of distance sampling surveys for estimating population size. J. Appl. Ecol., 47, 5-14.

Tutin C.E.G., White L.J.T. \& Mackanga-Missandzou A., 1997. The use by rain forest mammals of natural forest fragments in an Equatorial African savana. Conserv. Biol., 11(5), 1190-1203.

van Vliet N. \& Nasi R., 2007. Mise en évidence des facteurs du paysage agissant sur la répartition de la faune dans une concession forestière. Bois For. Trop., 292(2), 23-37.

van Vliet N. et al., 2007. Distinguishing dung from blue, red and yellow-backed duikers through noninvasive genetic techniques. Afr. J. Ecol., 46, 411-417.

van Vliet N. \& Nasi R., 2008. Why do models fail to assess properly the sustainability of duiker (Cephalophus spp.) hunting in central Africa? Oryx, 42(3), 392-399.

van Vliet N., Nasi R. \& Lumaret J.P., 2009. Factors influencing duiker dung decay in north-east Gabon: are dung beetles hiding duikers? Afr. J. Ecol., 47(1), 40-47.

van Vuuren B.J. \& Robinson T.J., 2001. Retrieval of four adaptive lineages in duiker antelope: evidence from mitochondrial DNA sequences and fluorescence in situ hybridization. Mol. Phylogenet. Evol., 20, 409-425.

Vanthomme H., Bellé B. \& Forget P.-M., 2010. Bushmeat hunting alters recruitment of large-seeded plant species in central Africa. Biotropica, 42, 672-679.

Viquerat S., Bobo K.S., Kiffner C. \& Waltert M., 2013. A comparison of regression-based estimates of dung decay in two African forest duiker species (Philantomba monticola, Cephalophus ogilbyi). Ecotropica, 19, 33-38.

Vrba E.S., 1995. The fossil record of African antelopes (Mammalia, Bovidae) in relation to human evolution and paleoclimate. In: Vrba E.S., Partridge T.C. \& Burckle L.H., eds. Paleoclimate and evolution with emphasis on human origins. New Haven, CT, USA: Yale University Press, 385-424.

White L.J.T., 1992. Vegetation history and logging disturbance: effects on rain forest mammals in the Lopé Reserve, Gabon (with special emphasis on elephants and apes). $\mathrm{PhD}$ thesis: University of Edimburg, Edimburg (Scotland).

White L.J.T., 1994. Biomasse des mammifères de forêt pluviale dans la réserve de la Lopé, Gabon. J. Anim. Ecol., 63, 499-512.

Yasuoka H., 2006. The sustainability of duiker (Cephalophus spp.) hunting for the baka hunter-gatherers in southeastern Cameroon. Afr. Study Monogr., Suppl., 33, 95-120.

Yasuoka H. et al., 2015. Changes in the composition of hunting catches in southeastern Cameroon: a promising approach for collaborative wildlife management between ecologists and local hunters. Ecol. Soc., 20(4), 25.

(59 réf.) 
Annexe 1. Caractéristiques descriptives des céphalophes des forêts denses humides d'Afrique centrale - Descriptive characteristics of duikers of central Africa rainforest (Groves et al., 2011 ; Kingdon \& Hoffmann, 2013 ; Castello, 2016).

\begin{tabular}{l} 
Espèces \\
\hline Cephalophus arrhenii \\
Cephalophus cataneus arrhenii Lönnberg, \\
1917 \\
Fr : Céphalophe d'Arrhenius \\
En : Uele white-bellied duiker \\
$\quad$ Eastern white-bellied duiker \\
Statut UICN : Préoccupation mineure \\
(sous C. leucogaster) \\
CITES : non inscrit
\end{tabular}

Cephalophus callipygus Peters, 1876

Fr : Céphalophe de Peters

En : Peter's Duiker

Statut UICN : Préoccupation mineure

CITES : non inscrit

\section{Description}

Longueur totale $105 \mathrm{~cm}$, queue $10,5 \mathrm{~cm}$ (mesure effectuée sur un seul individu),

hauteur aux épaules $42-51 \mathrm{~cm}$, poids $14,7-21 \mathrm{~kg}$. Les femelles pèsent environ $11 \%$ de plus que les mâles. L'espèce est légèrement plus grande que C.leucogaster. Le pelage est brun (plus vif que $C$. leucogaster), le ventre, la gorge et la croupe sont blanchâtres. Les épaules et les pattes antérieures sont grises, les jarrets des membres postérieurs sont marqués de poils noirs. L'espèce possède une bande dorsale noire de la même caractéristique que chez $C$. leucogaster, mais plus large. La largeur moyenne de cette bande dorsale est de 10,9 cm (à l'endroit le plus large). Les bords de la bande ont tendance à être diffus, apparaissant parfois dentelés. On observe une touffe prononcée de poils noirs et blancs à la pointe de la queue. Les parties faciales de l'espèce sont généralement rougeâtres, avec une bande noire qui va du pont du nez et se dilate sur le front. Les cornes sont courtes et pointues, avec des bases fortement striées. Chez les mâles, elles mesurent 2,1-6,9 cm et chez les femelles 1,8-2,9 cm.

Longueur totale 101,2-135 cm, queue 13-16,5 cm, hauteur aux épaules 50$57,6 \mathrm{~cm}$, poids $17,2-26,9 \mathrm{~kg}$. Les femelles sont généralement plus grandes que les mâles. L'ensemble du pelage peut varier du brun-roux au brun-foncé ou du fauve au roux. Une fine bande dorsale noire s'étend des épaules et longe la colonne vertébrale jusqu'aux pattes arrière, de sorte que l'ensemble de la croupe (y compris la queue) est noir. Les parties abdominales sont plus pâles que le reste du corps. On retrouve au-dessus de chaque œil deux taches blanches (0,8-1 cm de diamètre). On note la présence d'une touffe de poils roux audessus de la tête. Les oreilles sont blanches à leur surface intérieure et disposent d'une bande noire et blanche sur leurs bords. Les cornes sont courtes et pointues chez les deux sexes. Elles sont de 6,2-9,1 cm chez les mâles, 4-5,8 cm chez les femelles

\section{Cephalophus castaneus}

Cephalophus dorsalis castaneus Thomas, 1892

Fr : Céphalophe bai

En : Eastern bay duiker

Eastern black-backed duiker Statut UICN : Préoccupation mineure CITES : annexe II
Longueur totale 88,3-103,2 cm, queue 8,1-12,6 cm, hauteur aux épaules 45$52 \mathrm{~cm}$, poids $18,4-24,2 \mathrm{~kg}$. Les femelles sont légèrement plus grandes que les mâles. Le pelage est globalement brun rougeâtre. Les parties abdominales sont légèrement plus brillantes que le dos. Une large bande dorsale noire s'étend du cou à la croupe et se rétrécit par la suite en formant une ligne fine qui va jusqu'à la fin de la queue. La largeur de cette bande dorsale est généralement supérieure à $8 \mathrm{~cm}$, bien que dans certaines localités elle ne dépasse pas 4,6 cm. Les quatre pattes sont brun foncé. La queue est noire bordée de blanc et d'une touffe terminale blanche. La gorge est blanchâtre. Une tache blanche est habituellement présente au-dessus de chaque œil. Une bande frontale rougeâtre va du pont du nez pour former un large triangle sur le front. La tête porte une touffe brillante rouge-châtain. La face de l'animal et sa crête sont habituellement rouges. Les cornes des mâles sont plus longues que celles des femelles. Elles sont parallèles et lisses d'une base à l'autre et mesurent $5,1-9,3 \mathrm{~cm}$.

Longueur totale 96,5-104,1 cm, queue 13-16 cm, poids $20 \mathrm{~kg}$. Cephalophus crusalbum est plus petit que $C$. ogilbyi. Le pelage est brun doré. Les parties arrière et la croupe sont plus foncées que les côtés. Le ventre et le cou sont grisâtres. Une bande dorsale noire de 2,5-6 cm de largeur (plus large que C. ogilbyi) s'étend des épaules jusqu'à la croupe, où elle se rétrécit à $1 \mathrm{~cm}$ et va jusqu'à la pointe de la queue. Des poils noirs peuvent former une bande secondaire (jusqu'à $10 \mathrm{~cm}$ de largeur) près de la queue. Mis à part la bande médiane noire, la surface dorsale de la queue est brun doré. La face inférieure de la queue a des poils blancs plus longs. La queue porte également une longue touffe terminale gris-brun (environ $7,5 \mathrm{~cm}$ ). Les quatre pattes sont relativement longues et nettement blanches sous le carpe et le tarse. Une étroite bande brune se retrouve à l'avant des pattes, elle s'élargit et s'assombrit autour des sabots. La tête de l'espèce est grise, avec un front brun foncé, le museau est noir. Les cornes sont présentes chez les deux sexes, elles mesurent 8,7-10,9 cm pour les mâles et environ $5 \mathrm{~cm}$ pour les femelles. 
Annexe 1 (suite 1). Caractéristiques descriptives des céphalophes des forêts denses humides d'Afrique centrale - Descriptive characteristics of duikers of central Africa rainforest (Groves et al., 2011 ; Kingdon \& Hoffmann, 2013 ; Castello, 2016).

\begin{tabular}{l} 
Espèces \\
\hline Cephalophus hypoxanthus \\
Cephalophus nigrifrons hypoxanthus \\
Grubb et Groves, 2001 \\
Fr: Céphalophe d'Itombwe \\
En : Itombwe duiker \\
Statut UICN : En danger (sous \\
C. nigrifrons rubidus) \\
CITES : non inscrit
\end{tabular}

Cephalophus johnstoni Thomas, 1901

Fr : Céphalophe de Johnston

En : Johnston's duiker

Statut UICN : Préoccupation mineure

(sous $C$. weynsi)

CITES : non inscrit

Fr : Céphalophe à ventre blanc

En : Western white-bellied duiker Gaboon duiker

Statut UICN : Quasi menacé

CITES : non inscrit

\section{Description}

Peu de données disponibles. Données de mensuration probablement similaires à $C$. nigrifrons. Longueur totale $85-95 \mathrm{~cm}$, queue $11-15 \mathrm{~cm}$, poids $13-16 \mathrm{~kg}$. Le pelage est châtain tirant vers le jaune, il est distinctement plus pâle que C. nigrifrons. Il n'y a pas de bande dorsale noire. Cependant, la base des poils tire vers du noir. Les pattes sont légèrement plus foncées que le reste du corps. Le menton est blanc. Des cornes sont sans doute présentes chez les deux sexes.

Très peu d'informations disponibles sur cette espèce. Longueur totale $89,7 \mathrm{~cm}$ (mesure effectuée sur un seul spécimen), queue $10 \mathrm{~cm}$, poids inconnu (probablement inférieur à celui de $C$. weynsi). Pelage brun rougeâtre, devenant plus foncé aux épaules et au cou. Les parties abdominales sont brunes. Les pattes sont également brunes et s'assombrissent vers le sabot qui est noir. La queue est rousse (tendant vers le noir) à sa surface supérieure et blanche à sa surface inférieure. La touffe terminale de la queue présente un mélange de poils blancs et bruns. Le front quant à lui est recouvert d'un mélange de poils roux et noirs. Le museau est noir, les lèvres et le menton sont blancs. Les oreilles (dotées d'une bordure blanche) sont brunes à leur face extérieure et blanches à leur face intérieure. Les cornes sont longues de 8,8-10,7 cm chez les mâles et 3,5-3,7 cm chez les femelles.

Longueur totale $92-100 \mathrm{~cm}$, queue $12-15 \mathrm{~cm}$, hauteur aux épaules $42-51 \mathrm{~cm}$, poids $14-17,5 \mathrm{~kg}$. Les femelles ont tendance à être plus lourdes que les mâles (différence de $8 \%$ ). Les poils du céphalophe à ventre blanc sont courts sur tout le corps. Le pelage est globalement brun doré avec des tons rougeâtres. Cette coloration est plus intense le long du dos et vers la croupe. Les quatre pattes sont grises et noircissent vers les sabots. On retrouve des taches noires sur les jarrets. Les parties inférieures (la gorge, la poitrine, les surfaces ventrales, les fesses et les entrejambes) sont blanches. On distingue une bande dorsale noire partant de la nuque (ou des épaules) qui s'élargit au milieu du dos, puis se rétrécit ensuite jusqu'à la base de la queue. La bande dorsale est plus rétrécie que celle de $C$. arrhenii et peut présenter des bords irréguliers chez certains individus. Sa largeur va de 3,6 à $6,9 \mathrm{~cm}$. La queue est rougeâtre à sa surface supérieure et dénudée à sa surface inférieure. Elle dispose d'une touffe terminale de poils blancs avec des extrémités noires. La touffe de poils au-dessus de la tête est rousse et brillante, parfois mélangée avec des poils noirs. De courtes cornes très baguées se retrouvent chez les deux sexes. Elles sont en moyenne de 4,7 $\mathrm{cm}$ chez les mâles et $2,4 \mathrm{~cm}$ chez les femelles. Cependant, des cornes d'une longueur de 12,7 cm ont été enregistrées au Gabon.

Longueur totale $85-107 \mathrm{~cm}$, queue $10-16 \mathrm{~cm}$, hauteur aux épaules $53,5-57,8 \mathrm{~cm}$, poids 13-16 kg. Les femelles sont légèrement plus grandes que les mâles. La sous-espèce nigrifrons est généralement plus grande que la sous-espèce kivuensis. Le pelage dans son ensemble est châtain et brillant. Chaque poil a une base brune et un bout rougeâtre. Le pelage est épais, surtout chez kivuensis. On note une absence de bande dorsale noire. Toutefois, on observe une grande tache ou bande noire au niveau de la poitrine. Les pattes sont remarquablement longues. Leur coloration s'assombrit devenant presque noire aux sabots. Les sabots sont allongés et étroits. La queue est longue, noire avec une touffe terminée par des poils blancs. Les parties faciales sont colorées à l'identique du reste du corps. On y note une bande noire qui va du nez et englobe tout le front. Le front est plus noir chez kivuensis. Une fine bande rousse borde la bande noire du front au-dessus des yeux. Les oreilles sont noires, avec une tâche blanche à l'intérieur. Les cornes ont une longueur de 6,5-11,5 cm chez les mâles et 1,3-5,2 $\mathrm{cm}$ chez les femelles. 
Annexe 1 (suite 2). Caractéristiques descriptives des céphalophes des forêts denses humides d'Afrique centrale — Descriptive characteristics of duikers of central Africa rainforest (Groves et al., 2011 ; Kingdon \& Hoffmann, 2013 ; Castello, 2016 ).

\begin{tabular}{l} 
Espèces \\
\hline Cephalophus ogilbyi Waterhouse, 1838 \\
Fr : Céphalophe d'Ogilby \\
En : Ogilby's duiker \\
Statut UICN: Vulnérable (sous C. ogilbyi \\
ogilbyi)
\end{tabular}

CITES : annexe II (sous C. ogilbyi)

\section{Description}

Longueur totale $90 \mathrm{~cm}$, queue $15 \mathrm{~cm}$, hauteur aux épaules $56 \mathrm{~cm}$, poids 18 $20 \mathrm{~kg}$. Les pattes sont relativement longues. Le pelage est brun et fortement doré. La croupe a tendance à être très colorée. La nuque et les côtés du cou sont très poilus et plus bruns que le reste du corps. Une large zone de poils se retrouve sur la nuque. Des poils noirs s'étendent de la ligne médiane du cou et deviennent de plus en plus denses vers le bas en formant une bande dorsale noire bien définie qui va des épaules jusqu'à la base de la queue (chez certains individus, la bande n'est pas distincte des épaules jusqu'au milieu du dos). Cette bande est relativement étroite, de $1,1 \mathrm{à} 3 \mathrm{~cm}$ de largeur. Les pattes sont de la même couleur que le corps, mais elles s'assombrissent autour des sabots. Des bandes brun foncé peuvent être présentes à l'avant des pattes antérieures. La queue a une touffe terminale grise parfois assez grande. La touffe de poils au-dessus de la tête peut varier de l'orange clair au brun foncé. Des cornes légèrement ascendantes sont présentes chez les deux sexes. Chez les mâles, elles sont épaisses, avec des anneaux rugueux sur la moitié basale et peuvent être longues de $9 \mathrm{~cm}$. Celles des femelles ont environ $6 \mathrm{~cm}$ de long.

Cephalophus silvicultor

Antilope sylvicultrix Afzelius, 1815

Fr : Céphalophe à dos jaune

En : Western yellow-backed duiker

3 sous-espèces dont

C.s. longiceps et C. s. ruficrista

dans les forêts denses humides d'Afrique centrale

Statut UICN : Quasi menacé

CITES : annexe II
Longueur totale du corps $115-145 \mathrm{~cm}$, queue 11-18 cm, hauteur aux épaules $65-85 \mathrm{~cm}$, poids $45-80 \mathrm{~kg}$. Cephalophus silvicultor est la plus grande espèce de céphalophe. Le pelage est noir brunâtre et brillant, d'un aspect doux et huileux. Les pattes peuvent être légèrement plus foncées. Les parties abdominales (y compris la région inguinale) sont également noires. On note particulièrement au niveau de la colonne vertébrale de l'espèce une bande triangulaire de poils jaunes érectiles qui s'élargit vers la croupe. L'étendue de la bande jaune est variable selon les individus. Cependant, les poils à ce niveau sont toujours plus longs que les poils noirs adjacents (environ 6-7 $\mathrm{cm}$ de long au niveau de la partie la plus large du triangle). La croupe a des poils très courts et tend à être plus pâle par rapport au reste du corps. Elle peut être bordée de poils jaunes. La queue est courte et mince, avec une petite touffe noire à l'extrémité. Les lèvres sont blanches. Les oreilles sont noires à l'extérieur et bordées de poils blancs à l'intérieur. Une touffe de poils (5-8 cm de long) rousse ou orange est présente au sommet de la tête et entoure généralement les bases des cornes. Les cornes sont présentes chez les deux sexes. Elles sont des pointes lisses et fines qui s'inclinent vers l'arrière du crâne. La longueur maximale enregistrée est de 21,3 cm.

Cephalophus weynsi Thomas, 1901

Fr : Céphalophe de Weyns

En : Weyns's duiker

Statut UICN : Préoccupation mineure

CITES : non inscrit spécimens de la Forêt de l'Ituri, RD Congo), poids 14-17,5 kg. Le pelage est
Longueur totale $94-100,3 \mathrm{~cm}$, queue 12,7-20,3 cm (mesures effectuées sur trois châtain ou ocre délavé. L'abdomen est plus pâle que le reste du corps et l'aine est blanche. L'espèce ne possède pas une bande dorsale ou ventrale distincte. Toutefois, on note une zone noire au milieu du dos. La queue a une ligne noire à sa surface supérieure. On observe des stries blanches aux parties intérieures des pattes antérieures. Le museau est brun noir ou chocolat et la ligne médiane du visage est noire. Le front présente un mélange de poils noirs et rouges. Les oreilles sont courtes, arrondies et blanches aux extrémités. Les cornes sont présentes chez les deux sexes. Les cornes des femelles sont nettement plus courtes. Les bases des cornes sont gonflées. Longueur des cornes : 8,7-11,1 cm chez les mâles, 3,3-5,5 cm chez les femelles.

Données de mensuration insuffisantes. Longueur totale $60-67 \mathrm{~cm}$, queue $8-12 \mathrm{~cm}$, oreille $4,7-5,6 \mathrm{~cm}$ (pour les deux sexes, Ouganda). La mesure du crâne indique que les femelles sont légèrement plus grandes que les mâles. Le pelage est globalement brun terne. La surface dorsale a tendance à être plus foncée que les flancs. Une tache noire est présente de chaque côté des hanches supérieures vers la base de la queue. La transition entre les hanches (pâles) et la croupe (plus foncée) est relativement forte mais moins que celle de $P$. congica et P. melanorhea. Les parties abdominales sont gris pâle. Les cornes sont toujours présentes chez les mâles et tendent à être assez longues. La présence de cornes chez les femelles est très variable. 
Annexe 1 (suite 3). Caractéristiques descriptives des céphalophes des forêts denses humides d'Afrique centrale - Descriptive characteristics of duikers of central Africa rainforest (Groves et al., 2011 ; Kingdon \& Hoffmann, 2013 ; Castello, 2016).

\begin{tabular}{ll}
\hline Espèces & Description \\
\hline Philantomba anchietae & Longueur totale $60-67 \mathrm{~cm}$, queue $7-11 \mathrm{~cm}$, oreille $6 \mathrm{~cm}$, poids $5 \mathrm{~kg}$. Sur base des \\
Cephalophus anchietae Bocage, 1879 & mesures du crâne, c'est l'une des plus grandes espèces du genre Philantomba. \\
Fr : Céphalophe d'Angola & Les individus des zones côtières ont tendance à être plus grands que ceux des \\
En : Angolan blue duiker & terres intérieures. Le pelage de l'espèce est pâle ou brun-gris, s'assombrissant \\
Statut UICN : Préoccupation mineure & au niveau des surfaces dorsales (typique au genre Philantomba) jusqu'à la base \\
(sous P. monticola) & de la queue. La queue est noire à sa surface supérieure et blanche en dessous. \\
CITES : annexe II (sous P. monticola) & Les côtés sont gris pâle, tirant vers le rouge au niveau des hanches postérieures. \\
& Les pattes sont rouge-brun (pâle). Les cornes sont généralement présentes chez \\
& les deux sexes mais peuvent être absentes chez les femelles. Leur longueur \\
& moyenne est de 4,1-4,6 cm chez les mâles et de $2,6 \mathrm{~cm}$ chez les femelles.
\end{tabular}

Philantomba congica

Longueur totale $56-66 \mathrm{~cm}$, queue $8 \mathrm{~cm}$, hauteur aux épaules $32-38 \mathrm{~cm}$, poids Cephalophus nyasae congicus Lönnberg, 3,9-5 kg chez le mâle et 4,2-6,1 kg chez la femelle. Le pelage est globalement 1908

Fr : Céphalophe du Congo

En : Western blue duiker

Congo blue duiker

Statut UICN : Préoccupation mineure

(sous P. monticola)

CITES : annexe II (sous P. monticola) gris-marron pâlissant au gris sur les flancs. Le dos est plus foncé. Une bande horizontale noire distincte traverse les hanches jusqu'à la base de la queue, contrastant remarquablement avec la croupe et les cuisses qui sont d'une couleur brune très pâle. Les parties abdominales sont blanchâtres. La queue est noire au-dessus et blanche en dessous. Les pattes sont grises ou brun-gris. Des marques noires sont présentes au-dessus des sabots. L'espèce a une face noire avec des joues tirant vers le rouge. Des cornes simples semblables à des pointes sont présentes chez les deux sexes. Elles sont généralement de 2,8-4,5 cm chez le mâle et de 2,4-3 cm chez la femelle.

Philantomba defriesi

Longueur totale $54-56 \mathrm{~cm}$, queue $8 \mathrm{~cm}$, poids $5,4 \mathrm{~kg}$ (mâles) et 7,3 $\mathrm{kg}$ (femelles). Cephalophus nyasae defriesi Rothschild, 1904

Fr : Céphalophe De Fries

En : Zambia blue duiker

Statut UICN : Préoccupation mineure (sous P. monticola)

CITES : annexe II (sous P. monticola)

Poids le plus important du genre Philantomba. Le pelage est généralement gris-brun, bien qu'il existe des variations considérables entre les individus. Les pattes sont nettement rousses. Les surfaces avant des pattes ont tendance à être plus brillantes. Les régions abdominales sont blanchâtres et celles axillaires sont blanches et brillantes. La face de l'animal est marquée d'une bande foncée qui va du museau jusqu'au sommet de la tête. Une large séquence de poils roux se retrouve autour des yeux et borde la bande foncée. Les joues sont plus pâles que le reste du corps. Les oreilles portent à leur surface extérieure des poils blancs dispersés. La surface intérieure des oreilles est blanche. Les cornes sont présentes chez les deux sexes, en moyenne $4,4 \mathrm{~cm}$ chez les mâles et $3,1 \mathrm{~cm}$ chez les femelles. Elles sont largement espacées et ont tendance à avoir une légère courbure.

Philantomba melanorhea

Cephalophus melanorheus Gray, 1846

Fr: Céphalophe de Bioko

En : Bioko blue duiker

Statut UICN : Préoccupation mineure (sous P. monticola)

CITES : annexe II (sous P. monticola)
Longueur totale $50-59 \mathrm{~cm}$, queue 6-10 $\mathrm{cm}$, oreille 4,3-5,3 $\mathrm{cm}$, poids $3,9-5 \mathrm{~kg}$. Espèce généralement plus petite que $P$. congica . Le pelage est gris-marron avec des parties nettement foncées. Les poils sont longs et épais. Les flancs sont gris-rosâtre et les parties abdominales sont blanchâtres. La couleur noir du dos se répand jusqu'à la base de la queue pour former une bande horizontale. La surface dorsale de la queue est noire et celle inférieure est blanche. La face de l'animal et les pattes ont une couleur semblable à celle du corps. Les cornes sont présentes chez les deux sexes. Elles sont relativement longues, en moyenne $4,31 \mathrm{~cm}$ chez le mâle et $3,35 \mathrm{~cm}$ chez la femelle.

Longueur totale $60 \mathrm{~cm}$, queue $10 \mathrm{~cm}$, poids $5 \mathrm{~kg}$. Le céphalophe bleu de Simpson est le plus petit du genre Philantomba. Le pelage est rougeâtre. La tête, le cou et le corps sont brun rougeâtre et se foncent en brun-noir le long de la ligne médiane du dos. Les parties abdominales sont blanchâtres. Les pattes sont rouge-brun. Les hanches sont plus rouges que le reste du corps. La transition entre la partie dorsale plus foncée et les pattes arrière (rouges) est peu marquée. Des cornes sont généralement présentes chez les deux sexes. Elles peuvent cependant être absentes chez les femelles.

Lorsqu'il y a deux noms, le premier se réfère à la nouvelle nomenclature proposée par Groves et al. (2011) et Castello (2016) - When there are two names, the first one refers to the new nomenclature proposed by Groves et al. (2011) and Castello (2016). 
Annexe 2. Fruits consommés par six espèces de céphalophes sur base de trois études menées au nord-est du Gabon (classification APG III) - Fruits consumed by six duikers species based on three studies conducted in northeastern Gabon (APG III system) (Dubost, 1984 ; Feer, 1989a ; Feer, 1995).

\begin{tabular}{|c|c|c|c|c|c|c|}
\hline Espèce végétale & $\begin{array}{l}\text { Philantomba } \\
\text { congica }\end{array}$ & $\begin{array}{l}\text { Cephalophus } \\
\text { callipygus }\end{array}$ & $\begin{array}{l}\text { Cephalophus } \\
\text { castaneus }\end{array}$ & $\begin{array}{l}\text { Cephalophus } \\
\text { silvicultor }\end{array}$ & $\begin{array}{l}\text { Cephalophus } \\
\text { leucogaster }\end{array}$ & $\begin{array}{l}\text { Cephalophus } \\
\text { nigrifrons }\end{array}$ \\
\hline \multicolumn{7}{|l|}{ ANACARDIACEAE } \\
\hline Antrocaryon klaineanum Pierre & $\mathrm{X}$ & $\mathrm{X}$ & $\mathrm{X}$ & & $\mathrm{X}$ & \\
\hline Pseudospondias longifolia Engl. & $\mathrm{X}$ & $\mathrm{X}$ & $\mathrm{X}$ & $\mathrm{X}$ & $\mathrm{X}$ & $\mathrm{X}$ \\
\hline Tricoschypha spp. & & $\mathrm{X}$ & $\mathrm{X}$ & & & \\
\hline Indéterminée (1 espèce) & $\mathrm{X}$ & & & & & \\
\hline
\end{tabular}

\section{ANNONACEAE}

Anonidium mannii (Oliv.) Engl.

X

\& Diels

Artabotrys le-testui Pellegr. $\quad \mathrm{X} \quad \mathrm{X}$

Artabotrys congolensis De Wild.

\& T.Durand

$\mathrm{X}-\mathrm{X} \quad \mathrm{X}$

X

X $\quad X \quad$ X $\quad$ X

Annickia polycarpa (DC.) Setten $\mathrm{X}$

\& Maas ex I.M. Turner

Monanthotaxis sp.

$\mathrm{X}$

Duguetia confinis (Engl. \& $\quad$ X $\quad$ X

Diels) Chatrou

Greenwayodendron suaveolens $\quad \mathrm{X} \quad \mathrm{X}$

(Engl. \& Diels) Verdc.

X $\quad \mathrm{X} \quad \mathrm{X}$

$\mathrm{X}$

X

X

X

Uvaria klaineana Engl. \& Diels

Xylopia hypolampra Mildbr. X

Xylopia staudtii Engl. \& Diels

X $\mathrm{X}$ X

X X X

Indéterminées (7 espèces)

X $\mathrm{X} \quad \mathrm{X}$

APOCYNACEAE

Cylindropsis parvifolia Pierre

X X X

Dictyophleba stipulosa (S.Moore ex Wernham) Pichon

Indéterminées (27 espèces)

$\mathrm{X}$

X $\quad X \quad X$

APTANDRACEAE

Ongokea gore (Hua) Pierre

$\mathrm{X}$

$\mathrm{X} \quad \mathrm{X}$

ARECACEAE

Ancistrophyllum sp.

$\mathrm{X} \quad \mathrm{X}$

Calamus deerratus G.Mann \&

H.Wendl.

Eremospatha wendlandiana

Dammer ex Becc.

Eremospatha $\mathrm{sp}$.

$\mathrm{X}$

Indéterminées (4 espèces)

$\mathrm{X}$

X X X

X

X

ASPARAGACEAE

Dracaena arborea (Willd.) Link

X 
Annexe 2 (suite 1). Fruits consommés par six espèces de céphalophes sur base de trois études menées au nord-est du Gabon (classification APG III) - Fruits consumed by six duikers species based on three studies conducted in northeastern Gabon (APG III system) (Dubost, 1984 ; Feer, 1989a ; Feer, 1995).

\begin{tabular}{|c|c|c|c|c|c|c|}
\hline Espèce végétale & $\begin{array}{l}\text { Philantomba } \\
\text { congica }\end{array}$ & $\begin{array}{l}\text { Cephalophus } \\
\text { callipygus }\end{array}$ & $\begin{array}{l}\text { Cephalophus } \\
\text { castaneus }\end{array}$ & $\begin{array}{l}\text { Cephalophus } \\
\text { silvicultor }\end{array}$ & $\begin{array}{l}\text { Cephalophus } \\
\text { leucogaster }\end{array}$ & $\begin{array}{l}\text { Cephalophus } \\
\text { nigrifrons }\end{array}$ \\
\hline \multicolumn{7}{|l|}{ BALANOPHORACEAE } \\
\hline Thonningia sanguinea Vahl & & $\mathrm{X}$ & $\mathrm{X}$ & & $\mathrm{X}$ & \\
\hline \multicolumn{7}{|l|}{ BURSERACEAE } \\
\hline Canarium schweinfurtii Engl. & $X$ & $\mathrm{X}$ & $X$ & & & \\
\hline $\begin{array}{l}\text { Dacryodes buettneri (Engl.) } \\
\text { H.J.Lam }\end{array}$ & $X$ & $\mathrm{X}$ & $X$ & & $\mathrm{X}$ & $X$ \\
\hline $\begin{array}{l}\text { Dacryodes igaganga Aubrév. \& } \\
\text { Pellegr. }\end{array}$ & & & & & & $X$ \\
\hline $\begin{array}{l}\text { Dacryodes klaineana (Pierre) } \\
\text { H.J.Lam }\end{array}$ & & $\mathrm{X}$ & $\mathrm{X}$ & & & \\
\hline $\begin{array}{l}\text { Dacryodes normandii Aubrév. \& } \\
\text { Pellegr. }\end{array}$ & & $\mathrm{X}$ & & & & \\
\hline Santiria trimera I (Oliv.) Aubrév. & & $\mathrm{X}$ & $\mathrm{X}$ & & $\mathrm{X}$ & \\
\hline Santiria trimera II & & $\mathrm{X}$ & $X$ & & & \\
\hline Indéterminée (1 espèce) & $X$ & & & & & \\
\hline
\end{tabular}

CALOPHYLLACEAE

Mammea africana Sabine

$\mathrm{X}$

$\mathrm{X}$

X

$\mathrm{X}$

CAPPARIDACEAE

Indéterminée (1 espèce)

X

CELASTRACEAE

Salacia sp. (5 espèces)

$\mathrm{X}$

$\mathrm{X}$

CHRYSOBALANACEAE

Maranthes glabra (Oliv.) Prance

$\mathrm{X}$

CLUSIACEAE

Symphonia globulifera L.f.

$\mathrm{X}$

Indéterminées ( 2 espèces)

$\mathrm{X}$

\section{CONNARACEAE}

Rourea myriantha Baill.

X

Cnestis sp.

X

Indéterminées (4 espèces)

$\mathrm{X} \quad \mathrm{X}$

CONVOLVULACEAE

Neuropeltis acuminata (P.Beauv.)

X

Benth.

COULACEAE

Coula edulis Baill.

X

CUCURBITACEAE

Indéterminée (1 espèce)

EBENACEAE

Diospyros crassiflora Hiern

X $\quad \mathrm{X}$ 
Annexe 2 (suite 2). Fruits consommés par six espèces de céphalophes sur base de trois études menées au nord-est du Gabon (classification APG III) - Fruits consumed by six duikers species based on three studies conducted in northeastern Gabon (APG III system) (Dubost, 1984 ; Feer, 1989a ; Feer, 1995).

\begin{tabular}{|c|c|c|c|c|c|c|}
\hline Espèce végétale & $\begin{array}{l}\text { Philantomba } \\
\text { congica }\end{array}$ & $\begin{array}{l}\text { Cephalophus } \\
\text { callipygus }\end{array}$ & $\begin{array}{l}\text { Cephalophus } \\
\text { castaneus }\end{array}$ & $\begin{array}{l}\text { Cephalophus } \\
\text { silvicultor }\end{array}$ & $\begin{array}{l}\text { Cephalophus } \\
\text { leucogaster }\end{array}$ & $\begin{array}{l}\text { Cephalophus } \\
\text { nigrifrons }\end{array}$ \\
\hline \multicolumn{7}{|l|}{ ERYTHROPALACEAE } \\
\hline Heisteria parvifolia $\mathrm{Sm}$. & $\mathrm{X}$ & $\mathrm{X}$ & $\mathrm{X}$ & $\mathrm{X}$ & $\mathrm{X}$ & \\
\hline \multicolumn{7}{|l|}{ EUPHORBIACEAE } \\
\hline $\begin{array}{l}\text { Alchornea cordifolia (Schumach. \& } \\
\text { Thonn.) Müll.Arg. }\end{array}$ & $\mathrm{X}$ & $\mathrm{X}$ & & & & $\mathrm{X}$ \\
\hline $\begin{array}{l}\text { Maprounea membranacea Pax \& } \\
\text { K.Hoffm. }\end{array}$ & & $\mathrm{X}$ & & & & \\
\hline $\begin{array}{l}\text { Plagiostyles africana (Müll.Arg.) } \\
\text { Prain }\end{array}$ & & $\mathrm{X}$ & $X$ & & $\mathrm{X}$ & $\mathrm{X}$ \\
\hline $\begin{array}{l}\text { Ricinodendron heudelotii subsp. } \\
\text { Heudelotii }\end{array}$ & & $\mathrm{X}$ & $\mathrm{X}$ & $\mathrm{X}$ & & \\
\hline Indéterminées (3 espèces) & & & $\mathrm{X}$ & & $\mathrm{X}$ & \\
\hline
\end{tabular}

FABACEAE

Albizia dinklagei (Harms) Harms

X

Angylocalyx oligophyllus (Baker)

$\mathrm{X}$

Baker f.

Arachis hypogaea L.

Detarium macrocarpum Harms

Dialium dinklagei Harms

Dialium tessmannii Harms

Griffonia physocarpa Baill.

Pachyelasma tessmannii (Harms)

Harms

Parkia bicolor A.Chev.

Pentaclethra eetveldeana De Wild.

\& T.Durand

Pentaclethra macrophylla Benth.

Physostigma venenosum Balf.

Scorodophloeus zenkeri Harms

Indéterminées ( 2 espèces)

Indéterminée (1 espèce)

Indéterminées (31 espèces)

$\mathrm{X}$

$\begin{array}{cc} & X \\ X & X \\ X & X \\ & X \\ X & X \\ X & X\end{array}$

GENTIANACEAE

Anthocleista schweinfurthii Gilg

Anthocleista vogelii Planch.

X $\mathrm{X}$

\section{HUACEAE}

Afrostyrax lepidophyllus Mildbr.

X $\quad X$

\section{IRVINGIACEAE}

Irvingia gabonensis (AubryLecomte ex O'Rorke) Baill.

$\begin{array}{lllll}\text { X } & \text { X } & \text { X } & \text { X } & \text { X }\end{array}$

Irvingia grandifolia (Engl.) Engl.

X

$\mathrm{X}$

$\mathrm{X}$

X

X

X 
Annexe 2 (suite 3). Fruits consommés par six espèces de céphalophes sur base de trois études menées au nord-est du Gabon (classification APG III) - Fruits consumed by six duikers species based on three studies conducted in northeastern Gabon (APG III system) (Dubost, 1984 ; Feer, 1989a ; Feer, 1995).

\begin{tabular}{|c|c|c|c|c|c|c|}
\hline Espèce végétale & $\begin{array}{l}\text { Philantomba } \\
\text { congica }\end{array}$ & $\begin{array}{l}\text { Cephalophus } \\
\text { callipygus }\end{array}$ & $\begin{array}{l}\text { Cephalophus } \\
\text { castaneus }\end{array}$ & $\begin{array}{l}\text { Cephalophus } \\
\text { silvicultor }\end{array}$ & $\begin{array}{l}\text { Cephalophus } \\
\text { leucogaster }\end{array}$ & $\begin{array}{l}\text { Cephalophus } \\
\text { nigrifrons }\end{array}$ \\
\hline Klainedoxa gabonensis Pierre & $\mathrm{X}$ & $\mathrm{X}$ & $\mathrm{X}$ & $\mathrm{X}$ & $\mathrm{X}$ & $\mathrm{X}$ \\
\hline
\end{tabular}

\section{LAMIACEAE}

Vitex ferruginea Schumach. \&

$\mathrm{X}$

Thonn.

\section{LECYTHIDACEAE}

Petersianthus macrocarpus

$\mathrm{X}$

(P.Beauv.) Liben

Oubanguia africana Baill.

$\mathrm{X}$

Scytopetalum klaineanum Pierre ex

Engl.

\section{LINACEAE}

Hugonia planchonii Hook.f.

$\mathrm{X}$

Hugonia platysepala Welw. ex Oliv. X

$\mathrm{X}$

$\mathrm{X}$

$\mathrm{X}$

Hugonia spicata Oliv.

X $\quad X \quad X$

\section{LOGANIACEAE}

Strychnos aculeata Soler.

$\mathrm{X}$

Strychnos camptoneura Gilg \&

Busse

Strychnos ngouniensis Pellegr.

$\mathrm{X}$

\section{MALVACEAE}

Bombax buonopozense P.Beauv.

Cola rostrata K.Schum.

Duboscia macrocarpa Bocq.

Microcos barombiensis (K.Schum.)

$\mathrm{X}$

$\mathrm{X}$

$\mathrm{X}$

$\mathrm{X}$

Cheek

\section{MARANTACEAE}

Hypselodelphys violacea (Ridl.)

X

Milne-Redh.

\section{MELIACEAE}

Entandrophragma candollei Harms

$\begin{array}{ll} & X \\ X & X\end{array}$

Trichilia gilgiana Harms

$\begin{array}{ll} & X \\ X & X \\ X & X \\ X & \end{array}$

\section{MENISPERMACEAE}

Stephania sp.

$\mathrm{X}$

Indéterminée (1 espèce)

$\mathrm{X}$

$\mathrm{X}$

X

$\mathrm{X}$

\section{MORACEAE}

Ficus lingua Warb. ex De Wild. \&

T.Durand

$\mathrm{X}$

Ficus sp.

$\mathrm{X}$ 
Annexe 2 (suite 4). Fruits consommés par six espèces de céphalophes sur base de trois études menées au nord-est du Gabon (classification APG III) - Fruits consumed by six duikers species based on three studies conducted in northeastern Gabon (APG III system) (Dubost, 1984 ; Feer, 1989a ; Feer, 1995).

\begin{tabular}{|c|c|c|c|c|c|c|}
\hline Espèce végétale & $\begin{array}{l}\text { Philantomba } \\
\text { congica }\end{array}$ & $\begin{array}{l}\text { Cephalophus } \\
\text { callipygus }\end{array}$ & $\begin{array}{l}\text { Cephalophus } \\
\text { castaneus }\end{array}$ & $\begin{array}{l}\text { Cephalophus } \\
\text { silvicultor }\end{array}$ & $\begin{array}{l}\text { Cephalophus } \\
\text { leucogaster }\end{array}$ & $\begin{array}{l}\text { Cephalophus } \\
\text { nigrifrons }\end{array}$ \\
\hline \multicolumn{7}{|l|}{ MYRISTICACEAE } \\
\hline $\begin{array}{l}\text { Coelocaryon preussii Warb. or } \\
\text { Pycnanthus angolensis (Welw.) } \\
\text { Warb. }\end{array}$ & $X$ & $X$ & $X$ & & $X$ & $X$ \\
\hline $\begin{array}{l}\text { Pycnanthus angolensis (Welw.) } \\
\text { Warb. }\end{array}$ & & & $\mathrm{X}$ & & & \\
\hline $\begin{array}{l}\text { Scyphocephalium mannii (Benth. } \\
\text { \& Hook.f.) Warb. }\end{array}$ & $X$ & & & & & \\
\hline $\begin{array}{l}\text { Staudtia kamerunensis var. } \\
\text { gabonensis (Warb.) Fouilloy }\end{array}$ & $X$ & $X$ & $X$ & & $X$ & \\
\hline $\begin{array}{l}\text { Staudtia kamerunensis var. } \\
\text { gabonensis (Warb.) Fouilloy }\end{array}$ & & $X$ & $X$ & & & \\
\hline
\end{tabular}

\section{OCHNACEAE}

Lophira alata Banks X X X

ex C.F. Gaertn.

\section{PANDACEAE}

Panda oleosa Pierre

X $\quad X$

$\mathrm{X}$

\section{PHYLLANTHACEAE}

Margaritaria discoidea var. discoidea

Phyllanthus polyanthus Pax

$$
\mathrm{X}
$$

$\mathrm{X}$

$\mathrm{X}$

X

Uapaca guineensis Müll.Arg.

$\mathrm{X} \quad \mathrm{X}$

or Uapaca paludosa Aubrév. \&

Leandri

\section{PUTRANJIVACEAE}

Drypetes gossweileri S.Moore

Drypetes spinosodentata (Pax)

Hutch.

$\mathrm{X}$

Drypetes sp. (4 espèces)

$\begin{array}{ll}X & X \\ X & X\end{array}$

RHAMNACEAE

Ventilago africana Exell

$\mathrm{X} \quad \mathrm{X}$

\section{RHIZOPHORACEA}

Anopyxis klaineana (Pierre) Pierre $\mathrm{X}$ ex Engl.

\section{RUBIACEAE}

Massularia acuminata (G.Don)

$\begin{array}{ccc}\mathrm{X} & \mathrm{X} & \\ \mathrm{X} & \mathrm{X} & \mathrm{X} \\ \mathrm{X} & \\ \mathrm{X} & \end{array}$

Bullock ex Hoyle

Nauclea diderrichii (De Wild.) X

Merr.

Sarcocephalus pobeguinii Hua ex

Pobég.

Nauclea vanderguchtii (De Wild.)

E.M.A.Petit

$\mathrm{X}$

X $\quad$ X

$\mathrm{X}$

$\mathrm{X}$ 
Annexe 2 (suite 5). Fruits consommés par six espèces de céphalophes sur base de trois études menées au nord-est du Gabon (classification APG III) - Fruits consumed by six duikers species based on three studies conducted in northeastern Gabon (APG III system) (Dubost, 1984 ; Feer, 1989a ; Feer, 1995).

\begin{tabular}{|c|c|c|c|c|c|c|}
\hline Espèce végétale & $\begin{array}{l}\text { Philantomba } \\
\text { congica }\end{array}$ & $\begin{array}{l}\text { Cephalophus } \\
\text { callipygus }\end{array}$ & $\begin{array}{l}\text { Cephalophus } \\
\text { castaneus }\end{array}$ & $\begin{array}{l}\text { Cephalophus } \\
\text { silvicultor }\end{array}$ & $\begin{array}{l}\text { Cephalophus } \\
\text { leucogaster }\end{array}$ & $\begin{array}{l}\text { Cephalophus } \\
\text { nigrifrons }\end{array}$ \\
\hline $\begin{array}{l}\text { Oxyanthus speciosus subsp. mollis } \\
\text { (Hutch.) Bridson }\end{array}$ & & & $\mathrm{X}$ & & & \\
\hline Parapentas setigera (Hiern) Verdc. & & & & & & $\mathrm{X}$ \\
\hline Rytigynia sp. & $\mathrm{X}$ & & $\mathrm{X}$ & & & \\
\hline Sherbournia kiliotricha N.Hallé & & & $\mathrm{X}$ & & & \\
\hline $\begin{array}{l}\text { Sherbournia batesii (Wernham) } \\
\text { Hepper }\end{array}$ & & & $\mathrm{X}$ & & & \\
\hline Indéterminées (3 espèces) & & & $\mathrm{X}$ & & & $\mathrm{X}$ \\
\hline
\end{tabular}

\section{SALICACEAE}

Oncoba glauca (P.Beauv.) Planch. $\mathrm{X}$

\section{SAPINDACEAE}

Blighia welwitschii (Hiern) Radlk.

Eriocelum macrocarpum Gilg.

$\mathrm{X} \quad \mathrm{X}$

Pancovia turbinata Radlk.

$\mathrm{X} \quad \mathrm{X}$

Indéterminée (1 espèce)

$\mathrm{X}$

\section{SAPOTACEAE}

Baillonella toxisperma Pierre

Chrysophyllum beguei Aubrév. \& X

Pellegr.

Chrysophyllum lacourtianum

De Wild.

Chrysophyllum pruniforme Engl. $\quad \mathrm{X} \quad \mathrm{X}$

$\mathrm{X}-\mathrm{X}$

$\mathrm{X}$

$\mathrm{X}$

Omphalocarpum procerum

$\mathrm{X}$

P.Beauv.

Synsepalum longicuneatum

De Wild.

X $\quad$ X

$\mathrm{X}$

Indéterminées (2 espèces) $\quad$ X $\quad$ X

\section{SOLANACEAE}

Solanum torvum $\mathrm{Sw}$. $\mathrm{X}$

$\mathrm{X}$

\section{STROMBOSIACEAE}

Diogoa zenkeri (Engl.) Exell \& $\quad \mathrm{X}$

Mendonça

\section{URTICACEAE}

Musanga cecropioides $\mathrm{R}$.Br. ex $\quad \mathrm{X}$ X $\quad X$

$\mathrm{X}$

$\mathrm{X}$

Tedlie

Myrianthus arboreus P.Beauv.

X $\quad \mathrm{X}$

Indéterminée (1 espèce)

VITACEAE

Cissus dinklagei Gilg \& M.Brandt

\section{ZINGIBERACEAE}

Aframonum sp.

X

Indéterminée - undetermined : famille uniquement connue - family only known; $\mathrm{X}$ : espèces consommées - species consumed. 
Annexe 3. Rôle des céphalophes dans la dispersion des graines (classification APG III) - The role of duikers in seed dispersal (APG III classification) (Feer, 1995).

Espèce végétale

ANACARDIACEAE

Antrocaryon klaineanum Pierre

Pseudospondias longifolia Engl.

\section{BURSERACEAE}

Canarium schweinfurtii Engl.

\section{CALOPHYLLACEAE}

Mammea africana Sabine EUPHORBIACEAE

Ricinodendron heudelotii (Baill.) Heckel B

Drypetes sp.

B

C

B

Taille des graines

Philantomba congica Cephalophus Cephalophus
callipygus castaneus

Cephalophus silvicultor

\section{FABACEAE}

Detarium macrocarpum Harms

E

B

d

$\mathrm{n} / \mathrm{d}$

$\mathrm{n} / \mathrm{d}$

$\mathrm{d}$

$\mathrm{n} / \mathrm{d}$

d

\section{IRVINGIACEAE}

Irvingia gabonensis (Aubry-Lecomte ex C

O'Rorke) Baill.

Klainedoxa gabonensis Pierre

D

n

$\mathrm{n}$

$\mathrm{n} / \mathrm{d}$

$\mathrm{d}$

d

d

d

$\mathrm{n}$

n

$\mathrm{n} / \mathrm{d}$

\section{LAMIACEAE}

Vitex ferruginea Schumach. \& Thonn.

B

\section{LINACEAE}

Hugonia planchonii Hook.f.

B d

Hugonia platysepala Welw. ex Oliv.

B

Hugonia spicata Oliv.

A

n

$\mathrm{n} / \mathrm{d}$

$\mathrm{n} / \mathrm{d}$

d

C n

$\mathrm{n}$

$\mathrm{n}$

n

$\mathrm{n}$

\section{MENISPERMACEAE}

Stephania sp.

B

d

\section{RUBIACEAE}

Rytigynia sp.

A

d

d

\section{SAPOTACEAE}

Chrysophyllum beguei Aubrév. \& Pellegr. B

Chrysophyllum pruniforme Engl.

B

$\mathrm{n}$

d

d

p

\section{ESPÈCES INDÉTERMINÉES}

$\mathrm{N}^{\circ} 1$

B

d

d

$\mathrm{N}^{\circ} 2$

A

$\mathrm{N}^{\circ} 3$

A

d

d

$\mathrm{p}:$ prédateur - predator $; \mathrm{d}:$ disperseur - disperser $; \mathrm{n}:$ neutre - neutral $; \mathrm{A}:<1 \mathrm{~cm} ; \mathrm{B}: 1-2 \mathrm{~cm} ; \mathrm{C}: 2-3 \mathrm{~cm} ; \mathrm{D}: 3-4 \mathrm{~cm} ; \mathrm{E}$ :

$4-5 \mathrm{~cm}$. 